\title{
ECOULEMENTS A SURFACE LIBRE DE RÉVOLUTION GRADUELLEMENT VARIÉS
}

\author{
A. CRAYA \\ Ancien élève de l'Ecole Polytechnique
}

\section{GENERALITES}

Les écoulements que nous avons en vue dans ces lignes se rencontrent principalement dans les évacuateurs en puits.

Une grande portion, parfois la totalité de ces ouvrages est constituée par un radier fortement incurvé ; cette partie de l'écoulement où la courbure des filets joue un role important ne rentre pas dans notre propos, et l'on sait que son traitement théorique est difficile.

La région graduellement variée dont nous nous occupons ici n'existe que pour certains tracés et s'étend sur une certaine longueur à l'aval du seuil.

La raison d'ètre d'une telle entrée relativement plate est liée à diverses considérations et notamment la suivante : les données techniques, débit et hauteur de lame, fixent à peu près le diamètre du seuil ; par ailleurs une bonne

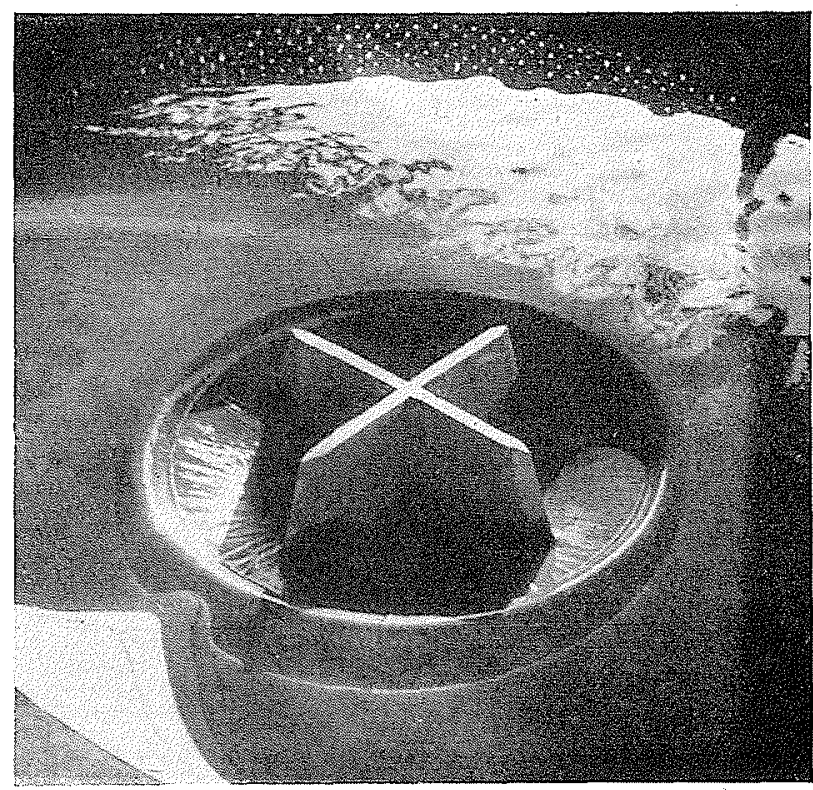

Photo 1 - ÉTUDE SUR MODÉLE RÉDUTY DE L'ÉVACUATEUil EN PUITS DU BARRAGE DE MONDEGO (PORTUGAL). IE CROISILLON CENTRAL EST UN DISPOSITIF ANTI-VORTEX. distribution de pressions sur le radier de l'ouvrage exige de ne pas trop s'éloigner de la forme que prendrait la nappe en jet libre ; si done le diamètre de départ se trouve trop grand, l'entonnoir en commençant immédiatement à cette dimension, risque de se raccorder mal ou pas du tout à un diamètre convenable de puits vertical ; il y a lieu alors de reporter la chute rapide un peu en aval et par conséquent de prévoir à l'entrée une zone à courbure modérée.

L'étude de cette zone, le voisinage du seuil excepté, ne fait appel qu'aux notions les plus simples de l'hydraulique des canaux; étant donné sa faible étendue, les pertes de charge de rugosité y sont négligeables et toute l'analyse peut être demandée au seul théorème de BErNoulu ; la seule particularité nouvelle par rapport aux canaux est la variation continue du débit spécifique (débit par unité de largeur); mais cette circonstance suffit à entraîner quelques aspects dignes de remarque qu'il a paru intéressant de signaler ici.

\section{LE DEVERSOIR CYLINDRIQUE}

Arrêtons-nous d'abord, à titre introductif, au cas d'un seuil ordinaire à bajoyers parallèles, (fig. 1).

L'évolution de la ligne d'eau est donnée, on le sait, par le diagramme très parlant de l'énergie spécifique ( $f i g .2$ ),

$$
\mathrm{E}=h+\frac{\mathrm{V}^{2}}{2 g}
$$

La distance de la ligne de charge au fond, donne en chaque section l'énergie spécifique disponible, d'où l'on déduit sur le graphique de $\mathrm{E}$ en fonction de $h$ la hauteur d'eau.

Partant de $A$, le point figuratif traverse l'état critique $C$ et décrit ensuite vers l'aval la branche supracritique CB .

Bien entendu, la notion d'énergie spécifique n'est applicable qu'à partir d'une certaine distance à l'aval de la crête où la courbure des' 
filets est peu sensible; si on étendait à tort le raisonnement précédent à tout le seuil, on serait conduit à situer la section critique sur la crête même et à définir le débit du déversoir ; en réalité, en raison des courbures et aussi de l'éventuelle perte de charge due à un décollement, ce débit échappe au calcul ; malgré ces restrictions, il sera commode quelquefois de parler de la section critique du déversoir ; il faudra entendre par là qu'en étendant vers l'amont le raisonnement de l'énergie spécifique avec le débit expérimental, on serait conduit à situer une section criticue en un point tel que $C$; ce point sera alor's caractéristique de l'écoulement en aval sans que la hauteur d'eau réelle y soit nćcessairement la hauteur critique.

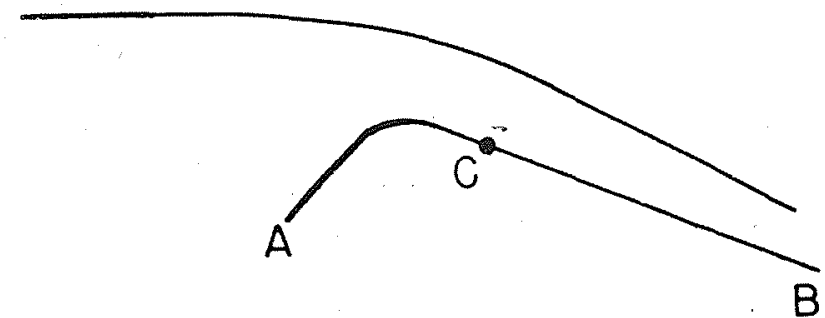

Fig. 1

L'emplacement de la section critique est en relation avec le coefficient de débit du déversoir donné par une relation du type :

$$
\eta=\frac{2}{3} \mathrm{CH} \sqrt{2 g \mathrm{H}}
$$

Le régime critique est en elfet tel que

$$
\mathrm{V}_{c}=\sqrt{g h_{c}}
$$

c'est-à-dire $\eta_{c}=h_{c} \sqrt{g h_{c}}$

et par suite $\mathrm{E}_{c}=\frac{3}{2} h_{c}=\frac{3}{2} \sqrt[3]{\frac{q_{c}}{g}}$

Compte tenu de la relation (1) on obtient :

$$
\mathrm{E}_{c}=\sqrt[3 \mathrm{C}^{2} \mathrm{H}]{ }
$$

Si l'on fait abstraction de toute perte de charge, il suffit de porter vers le bas à partir du niveau d'amont la distance (4) pour avoir la cote du radier de la section critique; pour des seuils à coefficient de débit élevé le point critique peut se trouver ainsi a un niveau au-dessous de la crête allant jusqu'à $20 \%$ de la hauteur H.

Faisons cependant une nouvelle restriction :

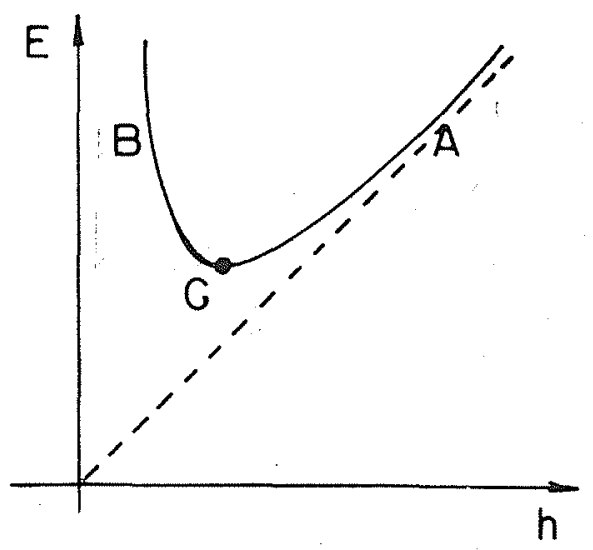

Fig. 2

la notion usuelle d'énergie spécifique suppose non seulement le courant graduellement varié mais encore les filets peu inclinés sur l'horizontale; il est d'ailleurs facile d'étendre la théorie aux courants fortement inclinés.

L'énergie spécifique n'est autre que la charge comptée à partir du fond d'une section; dans le cas d'une distribution de pression pseudohydrostatique de $\mathrm{M}$ en $\mathrm{N}$ ( $/ \ddot{i g} .3)$, tous les filets ont la même énergie :

$$
\begin{aligned}
\mathrm{E} & =h \cos \varphi+\frac{\mathrm{V}^{2}}{2 g} \\
\text { ou } \quad \mathrm{E} & =h^{\prime}+\frac{q^{\prime 2}}{2 g h^{\prime}}
\end{aligned}
$$

(en posant $h^{\prime}=h \cos \varphi \quad q^{\prime}=q \cos \varphi \quad \mathrm{et}$ $\left.\mathrm{U}=q^{\prime} / h^{\prime}\right)$

On voit qu'on peut étendre les propriétés du diagramme de la figure (2) à condition de remplacer $h$ par $h$ et $q$ par $q^{\prime}$, le régime critique correspond alors à :

$$
\mathrm{E}_{c}=\frac{3}{2} h_{c}^{\prime}=\frac{3}{2} \sqrt[3]{\frac{q_{c}^{\prime}}{g}}
$$

et la formule (4) doit s'écrire plus exactement :

$$
E_{c}=\sqrt[3]{3 C^{2} \cos ^{2} \varphi H}
$$

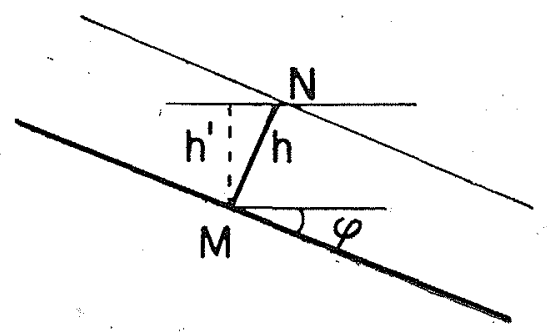

Fig. 3 
Sur un parement d'aval incliné le point critique se situerait done plus haut.

Notons au passage l'influence de la pente sur la vitesse critique, c'est-à-dire la célérité a des petites ondes ; celle-ci est donnce par la relation :

$$
a=\sqrt{g h}
$$

et elle est donc plus faible que pour un canal horizontal.

\section{DEVERSOIR DE REVOLUTION}

On peut appliquer aux seuils de révolution des considérations tout a fait analogues à celles qui préédent avec celte circonstance nouvelle que le débit spécificue varie d'une section à l'autre.

Si l'on se reporte au diagramme d'énergie spécifique (/ig. h) les courbes $\mathrm{E}(h)$ remontent en allant vers l'aval, el au lieł de décrire comme précédemment un arc CB c'est une ligne CC' qui figure maintenant l'écoulement dans les différentes sections.

Ici encore il peut être commode de caractériser le coefficient de débit du déversoir par la position d'une section critique d'entrée C ; c'est le point où serait réalisé l'état critique si l'on étendait vers l'amont (dans une zone où en général les courbures sont importantes) la ligne d'eau du courant graduellement varié d'aval.

Comme pour un déversoir plan, cette caractéristique dépend essentiellement du profil du seuil, dans une certaine zone au voisinage de la crête; partons d'un tel troncon et cherchons pour différents prolongements du radier en aval quelle est la ligne d'eau et éventuellement l'influence sur l'amont.

Le lieu des états critiques sur le diagramme $\mathrm{E}$ (h) est la droite OCC' ; pour qu'un débit donné puisse couler dans une section il faut une énergie spécifique minimum, qui est celle requise précisément par le régime critique; il n'y a pas d'écoulement possible, pour ce débit, avec une énergie moindre; si donc le tracé du radier en aval est tel, qu'à partir d'un certain point l'énergie spécifique disponible est inférieure à cette limite locale, cela veut dire que ce tracé réagit sur l'éconlement et que le débit n'est plus réglé par C mais par l'aval.

Considérons ( $i q g .5)$ un niveau amont déterminé et supposons que la section critique soit bien en $C^{\prime}$; soit $\Phi_{0}$ le débit spécifique, $\mathbb{E}_{o}$ l'énergie spécifique en $\mathrm{C}$.

$$
\mathrm{E}_{o}=\frac{3}{2} \sqrt[3]{\frac{g_{0}}{g}}
$$

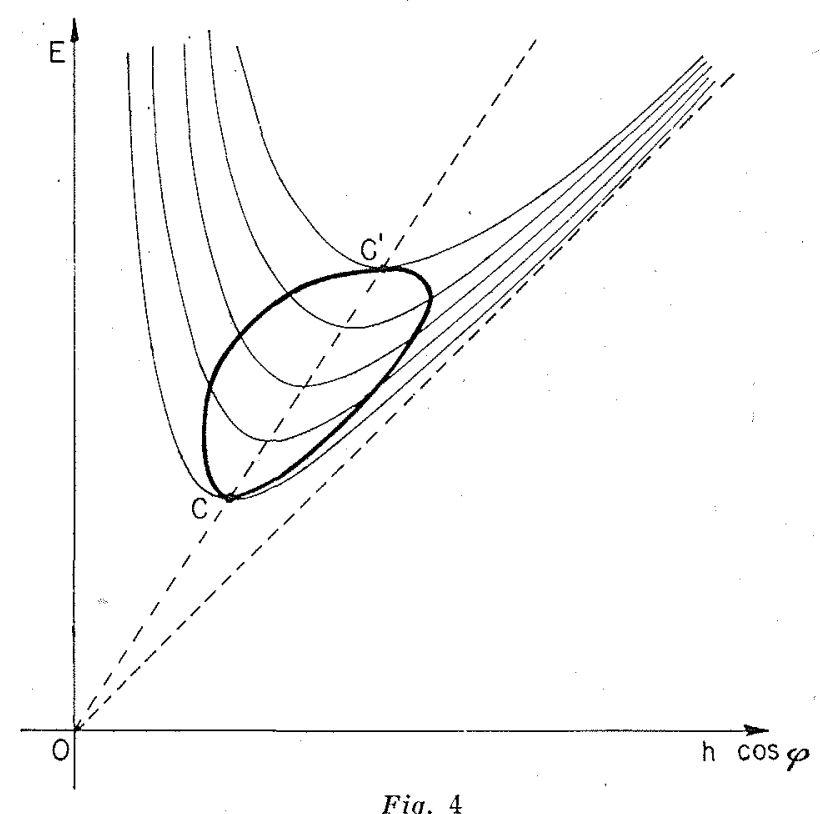

Dans toute autre section correspondant à un rayon $r$ le débit spécifique est donné par :

$$
\frac{q}{q_{0}}=\frac{r_{0}}{r}
$$

et l'énergie spécifique critique par :

$$
\frac{\mathrm{E}}{\mathrm{E}_{o}}=\left(\frac{r_{0}}{r}\right)^{2 \beta}
$$

D'après ce qui précède, si l'on ne veut pas que l'aval réagisse, pour cette charge d'eau, il y a toute une région interdite au radier : celle qui est située au-dessus de la courbe $\mathbf{E}$.

En adoptant pour méridienne la courbe $E$ elle-même le régime serait en toute section pour la charge $H$ le régime critique, c'est-à-dire que sur le diagramme d'énergie spécifique (fig. 4) on decrirait la droite $\mathrm{CC}^{\prime}$.

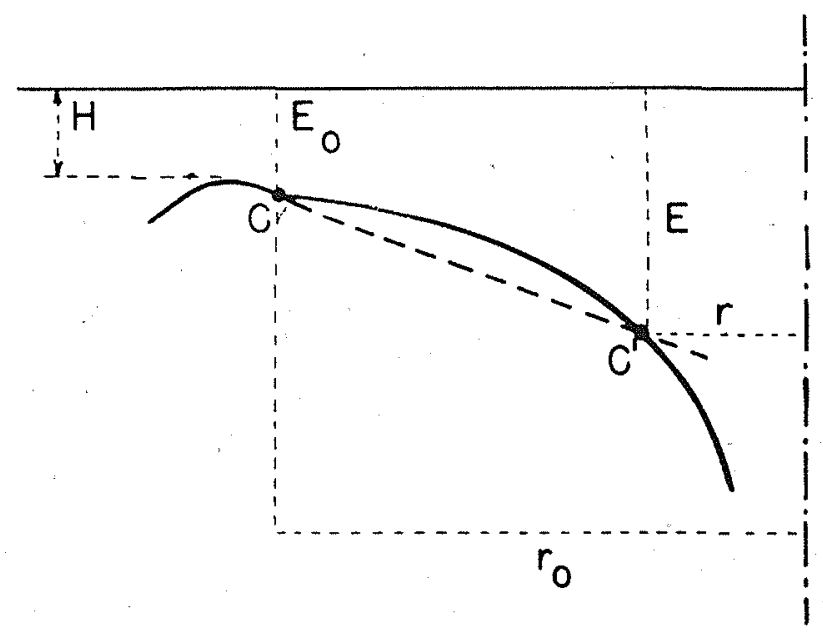

Fiq. 5 


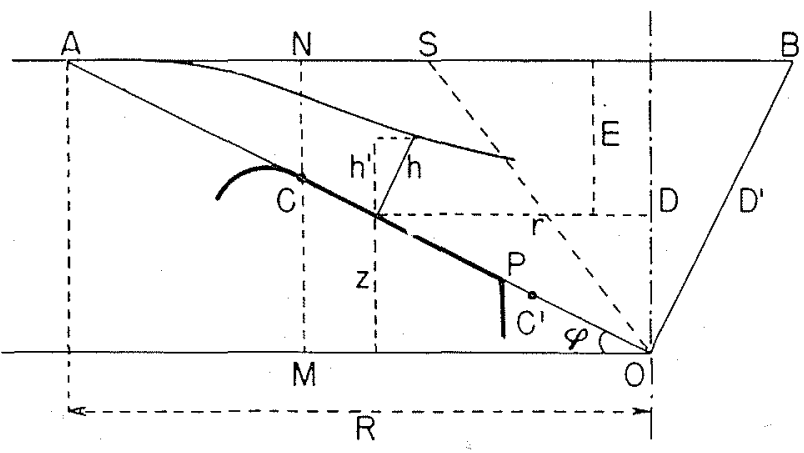

Fig. 6

Si cette méridienne traverse la courbe $\mathrm{E}$ en un autre point C' il y a embouteillage à l'aval et le débit est nécessairement moindre.

La ligne d'eau correspondant à la méridienne critique s'obtient aisément puisque la hauteur d'eau est alors les deux tiers de la charge; on voit que la profondeur augmente de l'amont à laval.

Dans ce qui précède on a négligé la pente du radier d'aval; si on en tient compte l'équation du profil eritique devient :

$$
\frac{\mathrm{E}}{\mathrm{E}_{0}}=\left(\frac{r_{0} \cos \varphi}{r^{\prime} \cos \varphi_{0}}\right)^{2 / 3}
$$

La courbe donnée par cette deuxième approximation se situe au-dessus de la première, laquelle demeure par conséquent une limite suffisante de sécurité; d'ailleurs là où la pente devient importante le courant n'est plus en général graduellement varié, de sorte que l'équation (5) ne mérite pas un calcul plus poussé.

Le radier limite dont il vient d'être question dépend de la charge $H$; mais il est clair que toutes ces courbes sont affines puisque

$$
\mathrm{E}-\mathrm{E}_{0}=\mathrm{E}_{0}\left[\left(\frac{r_{0}}{r}\right)^{9 / 3}-1\right]
$$

Par conséquent, si le radier est correctement. dimensionné pour ne pas engorger un débit maximum prévu, il en sera a fortiori de même pour tous débits inférieurs, résultat qui était évident à l'avance.

Ces résultats généraux étant ainsi précisès, nous allons maintenant calculer en détail l'écoulement sur un déversoir à méridienne rectiligne.

\section{ENTONNOIR CONIQUE}

Il est facile tout d'abord de calculer la posilion de la section critique $G$ (fig. 5) à partir de laquelle il faut abaisser le fond (ou élargir les bajoyers pour un partiteur) si l'on ne veut pas voir le débit diminuer.
Posant encore $h^{\prime}=h \cos \varphi$ (voir figure 6) et $q^{\prime}=q \cos \varphi$, nous avons vu que l'état critique vérifie :

$$
g h_{c}^{\prime}=q^{\prime 2} \quad \text { et } \quad h_{c}^{\prime}=\frac{2}{3} \mathrm{E}
$$

Le débit par unité de largeur $q$ est relié au débit Q qui s'écoule dans un dièdre d'angle $\alpha$ par :

$$
q=\frac{Q}{l \alpha}
$$

On a d'autre part :

$$
\mathrm{E}=\mathrm{D}-r \operatorname{tg} \varphi
$$

de sorte que l'équation domnant les sections critiques s'écrit :

$$
\frac{8}{27} g(\mathrm{D}-\lg \varphi)^{3}=\frac{\mathrm{Q}^{2} \cos ^{2} \varphi}{r^{2} \alpha^{2}}
$$

$$
\begin{gathered}
\text { Posant } \mathrm{R}=\frac{\mathrm{D}}{\operatorname{tg} \varphi} \text { et } \frac{r}{\mathrm{R}}=x \quad \text { il vient : } \\
x^{2}(1-x)^{*}=\frac{27}{8} \frac{\mathrm{Q}^{2} \sin ^{2} \varphi}{g \mathrm{D}^{3} \alpha^{2}}
\end{gathered}
$$

Résolution graphique immédiate en traçant la courbe

$$
y=x^{2}(1-x)^{3}
$$

(c'est la courbe I de la figure 7) et en la coupant par la droite $\mathrm{CC}^{\prime}$ d'ordonne convenable, ou, ce qui revient au même, en partant du point C d'amont.

L'abscisse de ce point critique d'amont est fig. (6)

$$
x_{0}=\frac{\mathrm{CM}}{\mathrm{CM}+\mathrm{CN}}
$$

Quand on part d'une charge faible, $x_{0}$ est voisin de l'unité, et le point critique conjugué

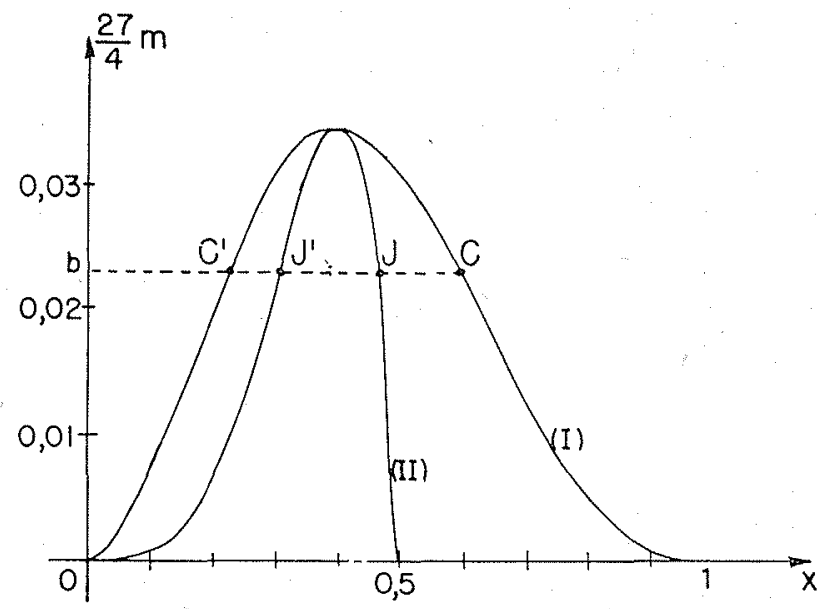

Fig. 7 
C' se situe tout près de l'axe de révolution : à mesure que la charge augmente le point $C^{\prime}$ remonte vers l'amont.

Considérons alors un entonnoir donné dont le seuil conique s'arrête au point $P$ par une chute brusque par exemple; tant que la charge sur le seuil est telle que le point critique conjugué C' tombe en aval de $\mathrm{P}$ le débit est bien réglé par le seuil en $C$; mais à partir du moment où le point C' tombe un peu en amont de $P$ (cette restriction en raison des courbures dans la zone C P) l'écoulement est commandé par le bord aval.

La charge limite à partir de laquelle cette circonstance se produit, se déduit aisément de la figure 7 ; il suffit de placer une droite $b$ C C' telle que le rapport $b \mathrm{C} / b \mathrm{C}$ ' soit égal à celui des rayons des points $C$ et $C$ ' sur le déversoir ; la relation (8) donne alors la valeur correspondante de $\mathrm{C} N$, c'est-à-dire le niveau amont limite.

Pour préciser davantage ce qui précède, on peut aussi calculer la forme de la surface libre. Celle-ci est donnée par le théorème de Bernot lli (fig. 6) :

$$
\dot{z}+h \cos \varphi+\frac{\mathrm{U}^{2}}{2 g}=\mathrm{D}
$$

Compte tenu de

$$
z=l \sin \varphi \text { et } \mathrm{U}=\frac{Q}{l \cos \varphi \alpha h}
$$

( $\alpha$ angle du dièdre) on obtient :

$$
l \frac{\sin \varphi}{\mathrm{D}}+h \frac{\cos \varphi}{\mathrm{D}}+\frac{\mathrm{Q}^{2}}{2 g \mathrm{D} \alpha^{2} \cos ^{2} \varphi \mathrm{L}^{2} l^{2}}=1
$$

Prenant enfin des valeurs relatives

$$
x=\frac{l}{\mathrm{~L}} \text { et } y=\frac{h}{\mathrm{D}^{\prime}} \text { où } \mathrm{L}=\mathrm{OA} \text { et } \mathrm{D}^{\prime}=\frac{\mathrm{D}}{\cos \varphi}
$$

Il vient :

$$
x+y+\frac{m}{x^{2} y^{2}}=1 \text { (9) avec } m=\frac{\mathrm{Q}^{2} \sin ^{2} \varphi}{2 g \mathrm{D}^{3} \alpha^{2}}
$$

On peut tracer aisément cette courbe en s'aidant par exemple d'un changement d'axe à $45^{\circ}$ des premiers; l'arc intéressant du point de vue hydraulique a été représenté sur la figure 8 ; il se divise lui-même en deux portions distinctes; la branche inférieure CKJC' (de l'amont à l'aval) est torrentielle et c'est celle qui nous intéresse principalement; la branche supérieure CJ'K'C' est fluviale et serait à considérer à l'aval d'un ressaut.

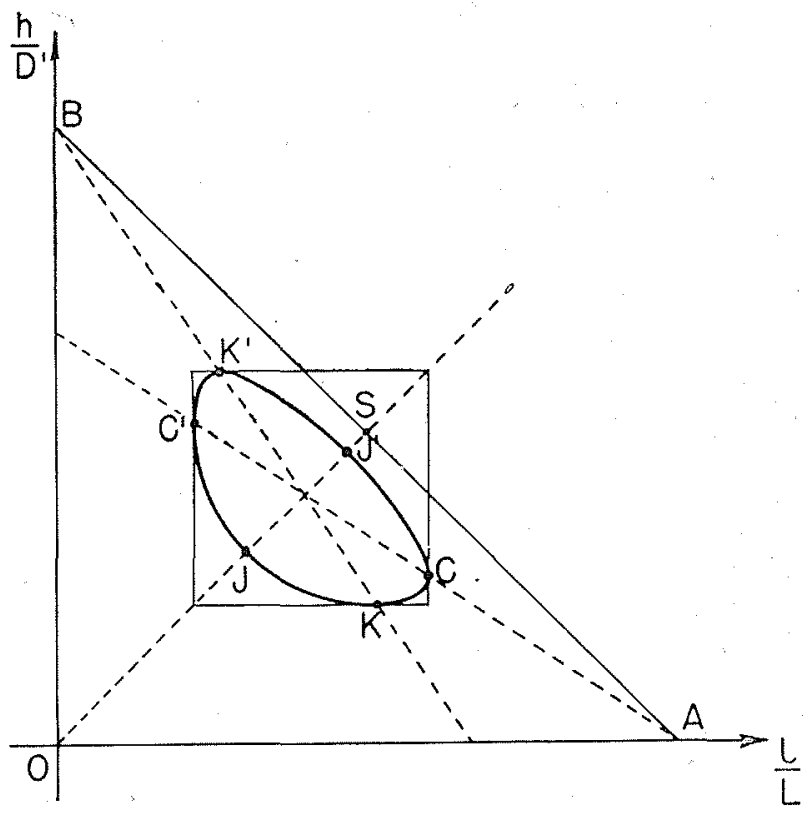

Fig. 8

On vérifie aisément que $C$ et $C^{\prime}$ sont les points critiques précédemment considérés. En effet, on déduit de l'équation (9)

$$
y=-\frac{y}{x} \frac{3 x+2 y-2}{2 x+3 y-2}
$$

et les points a tangente verticale de la figure 8 vérifient

$$
y=\frac{2}{3}(1-x)
$$

équation qui n'est autre que

$$
h_{c}^{\prime}=\frac{2}{3} \mathrm{E}_{c}
$$

La cuuse ciant symétrique par rapport à la première bissectrice, les points $K, K^{\prime}$ à tangente horizontale se déduisent immédiatement des précédents.

Pour la ligne de remous torrentielle, on voit que la hauteur ne décroît que dans une région restreinte $\mathrm{CK}$, tandis qu'en aval la hauteur' est essentiellement croissante; ceci est dû̀ à l'influence prépondérante de l'augmentation du débit spécifique à mesure qu'on descend vers l'aval; il y a lieu de noter aussi qu'en raison des courbures, la portion $\mathrm{CK}$ ne représente pas véritablement la ligne d'eau; aux alentour's de $K$ celle-ci se raccorde à la droite $A B$ qui sur la figure 8 représente le niveau d'amont.

On peut achever de déterminer très suffisamment la ligne de remous en calculant les points $J$ et $J$ ' qui se trouvent sur la bissectrice os 
de la figure 8 , laquelle correspond sur la figure 6 à la médiane $\mathrm{O} S \mathrm{~S}$ du triangle $\mathrm{A} O \mathrm{O}$.

Ces points vérifient :

$$
m=x^{4}(1-2 x)
$$

La courbe II de la figure 7 représente l'équation

$$
y_{2}=\frac{27}{4} x^{\prime}(1-2 x)
$$

L'intersection des courbes I et II avec la parallèle à $\mathrm{O} x$ d'ordonnée $\frac{27}{4} m$ donne ainsi simultanément les points CC' KK' et JJ'. Nous terminerons par une remarque relativement au trajet décrit par le point figuratif du régime sur le diagramme d'énergie spécifique (fig. 4.); ce lieu coincide avec la ligne de remous en variables relatives de la fig. 8 ; on a en effet :

$$
x=1-\frac{\mathrm{E}}{\mathrm{D}} \quad y=\frac{h \cos \varphi}{\mathrm{D}}
$$

Il suffit donc de faire pivoter la fig. 4 te $\frac{\pi}{2}$
ur retomber sur la fig. 8 .

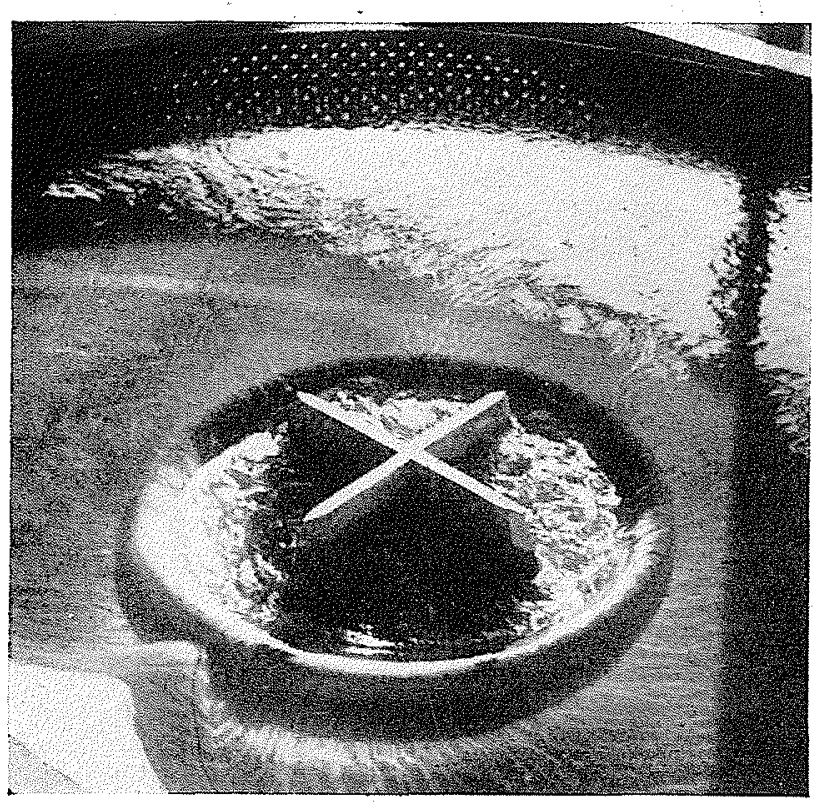

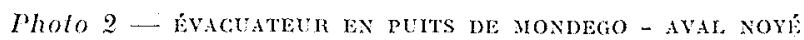

